\title{
Die EU-Verordnung 536/2014: Neuregulierung klinischer Prüfungen von Arzneimitteln - „Unabhängige, universitäre klinische Arzneimittelprüfungen - quo vadis?““
}

\section{Gerd Richter}

Online publiziert: 25. März 2015

(C) Springer-Verlag Berlin Heidelberg 2015

Am 27. Mai wurde die ,Verordnung (EU) Nr. 536/2014 über klinische Prüfungen mit Humanarzneimitteln“ im Amtsblatt der EU veröffentlicht. Diese gilt ab dem 16. Juni 2014 in allen EU-Ländern. Zielsetzung der Neuregulierung ist die Harmonisierung der Anforderung an klinische Arzneimittelstudien und die Entbürokratisierung des Genehmigungsverfahrens bei klinischen Studien in der EU. Mit Geltung dieser EU-Verordnung ist allerdings noch die Anpassung an nationale Regelungen und Abläufe zu tätigen, was in Deutschland Änderungen im Arzneimittelgesetz (AMG) und in der Verordnung über die Anwendung der Guten Klinischen Praxis bei der Durchführung von klinischen Prüfungen mit Arzneimitteln zur Anwendung am Menschen (GCP-Verordnung) notwendig macht. Darüber hinaus wird diese Neuregulierung erst bei erwiesener Funktionstüchtigkeit des neu zu etablierenden zentralen EU-Portals zur Einreichung von Anträgen klinischer Arzneimittelstudien auf Genehmigung in Kraft treten, damit ist voraussichtlich frühestens in der zweiten Hälfte des Jahres 2016 zu rechnen. Diese Neuregulierung ist nach der 12. AMG-Novelle aus 2004 die bedeutendste Änderung in Bezug auf die Durchführung von klinischen Arzneimittelprüfungen.

Die wesentlichen Änderungen sind:

- Akademische Forschungseinrichtungen oder Pharmaunternehmen stellen Genehmigungsanträge für klinische Prüfungen - egal ob monozentrisch oder multizentrisch, mononational oder multinational - über ein zentrales Online-Portal;

- multinationale klinische Prüfungen werden federführend von einem Mitgliedsstaat koordiniert, wobei weitere beteiligte Mitgliedsstaaten Mitspracherechte haben;

- Genehmigungs- und Bearbeitungsfristen der Behörden sind geringfügig verkürzt worden;

- kurze Fristen für Sponsoren;

Prof. Dr. med. G. Richter $(\bowtie)$

Ethikkommission FB Medizin, Philipps-Universität Marburg,

Baldingerstrasse 1,

35032 Marburg, Deutschland

E-Mail: richterg@staff.uni-marburg.de 
- minimalinterventionelle klinische Prüfungen werden erleichtert;

- das Prinzip der stillschweigenden Genehmigung wurde eingeführt, d. h. äußert sich eine Genehmigungsbehörde nicht in der entsprechenden Frist, gilt dies automatisch als Zustimmung im Sinne des Antragstellers;

- bei der Forschung an nicht einwilligungsfähigen Patienten ist auch gruppennützige Forschung zugelassen;

- die Ergebnisse aller klinischen Prüfungen müssen veröffentlicht werden.

Während die Harmonisierung, Entbürokratisierung und insbesondere die Veröffentlichung der Ergebnisse aller klinischen Prüfungen zu begrüßen sind, müssen allerdings gegenüber einigen der Änderungen kritische Vorbehalte ausgesprochen werden in Bezug auf die Ermöglichung einer von der Pharmaindustrie unabhängigen akademischen, universitären klinischen Arzneimittelforschung.

1. Die festgelegten Fristen für die Sponsoren, d. h. im universitären Kontext die Universitäten, universitäre Arbeitsgemeinschaften, akademische Investigatoren, betragen für die Behebung von formalen Mängeln im Genehmigungsverfahren zehn Kalendertage und für zusätzliche Informationen (inhaltliche Nachforderungen) lediglich zwölf Kalendertage. Bei Fristablauf bzw. Fristversäumnis bedeutet dies die automatische Rücknahme des Antrages!

Zwar kann eine Änderung und Neueinreichung jederzeit erneut erfolgen, allerdings bedeutet dies einen kompletten Neustart des Genehmigungsverfahrens inklusive aller Fristen und Kosten.

Bei der klinischen Arbeitsbelastung von vielen an klinischer Forschung interessierten Klinikern und Arbeitsgruppen ist eine so kurze Fristeinhaltung insbesondere bei inhaltlichen Nachforderungen (z. B. zum biometrischen Studiendesign und/oder zu den statistischen Auswertverfahren) kaum möglich, wohl auch kaum unter Hilfestellung und Kooperation mit den in Deutschland an den medizinischen Fakultäten gut etablierten Koordinierungszentren für klinische Studien.

Selbst wenn eine Neueinreichung problemlos möglich ist, werden große Schwierigkeiten in der dazu notwendigen Mittelbeschaffung für die mit dem erneuten Genehmigungsverfahren verbundenen Kosten entstehen.

In dieser Hinsicht können diese Punkte ein großes Problem in der unabhängigen, akademischen Durchführung von klinischen Arzneimittelstudien darstellen und müssen als ein ethisches Dilemma angesehen werden.

2. Minimalinterventionelle Prüfungen, denen Vorteile hinsichtlich von weniger strengen Regeln in Bezug auf die Überwachung, die Anforderungen an den Inhalt des Master Files und die Rückverfolgbarkeit der Prüfpräparate zugestanden werden, müssen per definitionem (Artikel 2, Abs. 2, Nr. 3 EU-Verordnung) alle der folgenden Bedingungen, ohne Ausnahme, erfüllen:

- Die Prüfpräparate müssen zugelassen sein, außer Placebos;

- dem Prüfplan der klinischen Prüfung entsprechend, müssen die Prüfpräparate gemäß den Bedingungen der Zulassungen verwendet werden oder es muss eine evidenzbasierte Verwendung vorliegen, die durch veröffentlichte wissenschaftliche Erkenntnisse über Sicherheit und Wirksamkeit dieser Prüfpräparate nachgewiesen sein müssen; 
- zusätzliche Diagnostik und Überwachungsverfahren dürfen nicht mehr als minimale zusätzliche Risiken oder Belastungen im Vergleich zur normalen klinischen Praxis darstellen.

Waren Therapieoptimierungsstudien in der Vergangenheit eine Domäne der universitären klinischen Arzneimittelprüfungen, so ist sehr fraglich, ob diese Studien überhaupt im Sinne der EU-Verordnung als minimalinterventionelle klinische Prüfungen einzustufen sind und in Zukunft noch an universitären Kliniken im Sinne unabhängiger klinischer Forschung durchgeführt werden können.

3. Darüber hinaus erscheint die Einbindung der universitären Ethikkommissionen, die an den medizinischen Fakultäten seit Jahrzehnten erfolgreich etabliert sind, schwierig aufgrund der Fristen und der Änderungen hinsichtlich der Neuerung, dass lediglich eine Ethikkommission (EK) in Zusammenarbeit mit der Bundesoberbehörde (BOB) als koordinierende und damit federführende Ethikkommission im Rahmen einer multizentrisch-multinationalen klinischen Prüfung verantwortlich ist für die Bewertung der klinischen Prüfung in allen beteiligten EU-Mitgliedsstaaten. Das bedeutet für die EK und die BOB, dass sie ihre Bewertung innerhalb von 45 Tagen mit einem Bewertungsbericht abschließen müssen. Dabei sind mehrere sequentielle Arbeitsschritte zu tätigen, die einen großen Aufwand in relativ kurzer Frist bedeuten:

- Beratung in der Ethikkommission (u. a. mittels gründlicher Literaturrecherche, interdisziplinärer Diskussion u. a. m.) und anschließende Erstellung eines gemeinsamen Berichtsentwurfs mit der BOB innerhalb von 26 Tagen,

- Durchführung eines koordinierenden Reviews des Berichtsentwurfs unter Mitwirkung aller beteiligten Mitgliedsstaaten (z. B. schriftlich und im Rahmen von Telefonkonferenzen) innerhalb von zwölf Tagen und

- finale Erstellung des Bewertungsberichts gemeinsam mit der BOB innerhalb von sieben Tagen.

Dies wird von vielen universitären Ethikkommissionen mit der vorhandenen personellen Ausstattung und IT-Ausstattung, die von der jeweiligen Fakultät bezahlt und damit zur Verfügung gestellt wird, kaum möglich sein. Zwar wird darauf gehofft, dass die Gebühren kostendeckend sein werden, allerdings ist dies nicht sicher und kaum zu erwarten. Hier ist nach der Verantwortung des Bundes und der Länder hinsichtlich der Sicherstellung einer Bundes- bzw. Länderaufgabe zu fragen.

Es stellt sich somit auch nach der Einführung der EU-Verordnung 536/2014 weiterhin die Frage: „Unabhängige, universitäre klinische Arzneimittelprüfungen - quo vadis?“. Eine einseitige Durchführung von klinischen Prüfungen jenseits der Universitäten und Medizinischen Fakultäten kann eigentlich nicht im Sinne der Gesetzgeber sein! 